\title{
Forces prediction in micro-grinding single-crystal copper considering the crystallographic orientation
}

\author{
Man Zhao ${ }^{1,2}$, Xia Ji ${ }^{1, *}$, Beizhi $\mathrm{Li}^{1}$, and Steven Y. Liang ${ }^{1,2}$ \\ ${ }^{1}$ School of Mechanical Engineering, Donghua University, Shanghai 201620, PR China \\ ${ }^{2}$ Woodruff School of Mechanical Engineering, Georgia Institute of Technology, Atlanta30332, USA
}

Received: 13 August 2018 / Accepted: 11 October 2018

\begin{abstract}
In the micro-grinding of single-crystal copper, the effect of crystallography becomes significant as the wheel works intra-crystalline. To quantify the effect of crystallographic orientation (CO) related to the cutting direction on the micro-grinding process, this article presents a Taylor factor model by examining the number and style of activated slip systems. Then, the flow stress model of monocrystalline material is developed considering the variation of the Taylor factor. Furthermore, the models of chip formation and rubbing forces are derived from the flow stress model, while the plowing force is predicted by the Vickers hardness. Then, the overall grinding force model of the whole wheel is developed by incorporating the process parameters and the wheel properties. Finally, micro-grinding experiments are conducted to verify the model, using only the Taylor factor as the variable. The proposed analysis is also compared with the previously reported model, which considers the Taylor factor as a constant of 3.06. The comparison between the two predictions and experimental data shows that the consideration of Taylor factor variability improves the accuracy of prediction.
\end{abstract}

Keywords: crystallographic orientation / Taylor factor / flow stress / Vickers hardness / force

\section{Introduction}

Within the mechanical micromachining process, microgrinding is commonly the final step of manufacturing parts. Micro-grinding force, as the main factor on the surface integrity of parts, deserves a comprehensive investigation by considering the process conditions, micro-grinding wheel properties, and the effect of microstructure. Understanding and controlling the micro-grinding force is necessary to improve the quality of parts' surface. Although a micro-grinding process resembles a traditional grinding process, this process is distinctive due to the size effect in micromachining.

In micromachining, the depth of cut is usually less than the grit edge radius; the specific cutting force increases with decreasing chip thickness, which is depicted as the size effect. Many researchers have investigated and explained the phenomenon in the past decades. Lucca et al. [1] and Fang [2] attributed size effect to the plowing effect of tool with high rake angle when the undeformed chip thickness is less than the tool edge radius. Some other investigations also contributed to the explanation of edge radius effects [3-5]. Nakayama and Tamura [6] attributed the size effect

\footnotetext{
* e-mail: jixia0206@163.com
}

to the subsurface plastic deformation and material strengthening effects. Atkins [7] and Subbiah and Melkote [8] cited material separation as the contributing factor to the size effect. Zhang et al. [9] investigated the size effect of specific cutting energy in mechanical micro-cutting. The results showed that the deformation coefficient of chip thickness and specific cutting energy increases with the ratio of uncut chip thickness to cutting edge radius decreasing due to the extruding of the round cutting edge. Chen et al. [10] analyzed the cutting surface quality in microball end milling considering size effect. The authors concluded that the surface roughness Ra decreases with the increasing of depth of cut for size effect points. The smaller undeformed chip thickness would intensify the plowing effect, and then increase the surface roughness. Liu et al. [11] experimentally investigated the size effect of tool edge on the chip formation. The result showed that the chip deformation increases sharply with the decrease in the depth of cut when the depth of cut is reduced to less than the tool edge radius. In the micro-grinding process, single grit interaction is similar to micromachining with a high negative rake angle. Shaw [12] conducted a Brinell indentation hardness test to demonstrate the single grit interaction and calculated the stress in the plowing region. Park and Liang [13] modeled the grinding force by considering plowing effect and the tool edge radius, based 
on the investigation that the deformation of material crushed by a Brinell ball resembled the behavior of material grinding beneath a grit. Except the size effect, the crystallographic effect becomes significant as the depth of cut is less than the average grain size of workpiece material in the micromachining process.

Both the crystallographic orientation and size have important effects on the micromachining processes. Hall [14] and Petch [15] presented the Hall-Petch relationship to quantify the effect of grain size on the flow stress of the polycrystalline material. Hughes and Hansen [16] further modified the flow stress model by adding the effect of dislocation density. However, in the model of the flow stress, the effect of $\mathrm{CO}$ is taken as a fixed value of 3.06 [17], which didn't consider the variation of CO. Numerous contributions have experimentally investigated the effect of $\mathrm{CO}$ on process behaviors, such as cutting force and surface finish. Ueda and Iwata [18] analyzed the effect of $\mathrm{CO}$ on the chip formation mechanism in the cutting of single-crystal $\beta$-brass. The investigations found that the shear angle, the cutting force, and surface integrity varied with the variation of cutting direction with respect to $\mathrm{CO}$ of workpiece material. Wu et al. [19] conducted a set of micromachining experiments on single-crystal copper materials to investigate the effect of material microstructures on cutting force. The results proved that CO has an essential impact on the cutting force and the burr formation. Lawson et al. [20] experimentally investigated the effect of crystallographic anisotropy on the orthogonal cutting of single-crystal aluminum. Li et al. [21] investigated the relationship between $\mathrm{CO}$ and micro-cracks of $\mathrm{Ti}-$ $47 \mathrm{Al}-2 \mathrm{Cr} 2 \mathrm{Nb}$ alloy. Results show that the $\mathrm{CO}$ is adverse in the nearly $\gamma$-microstructure and becomes advantageous in the fully lamellar microstructure. Xu et al. [22] investigated the effect of $\mathrm{CO}$ on the surface generation of aluminum in nano-cutting employing the molecular dynamics simulation. The authors concluded that the minimum uncut chip thickness is relatively small and the surface quality is better in the specific cutting direction. Cutting forces, chip morphology, shear stresses, shear angle, and effective coefficient of friction were seen to vary with CO. To investigate the effect of $\mathrm{CO}$ on micro-cutting force, Zhao et al. [23] presented a Taylor factor model to quantify the $\mathrm{CO}$ of cutting plane in the micro-cutting of monocrystalline material with a single grit. However, the cutting direction with respected to the $\mathrm{CO}$ is not considered in the Taylor factor model. In addition, the authors didn't calculate the overall force of the grinding wheel and the rubbing forces.

This article aims to propose a quantitative and physicsbased analysis about the effect of CO on the micro-grinding forces. The new Taylor factor model is proposed to examine the number and style of the activated slip systems, which are determined by the $\mathrm{CO}$ of machining plane and the cutting direction on the workpiece. In the predictive model of micro-grinding force, the Taylor factor contributes to the flow stress and then affects the chip formation and rubbing force, which contributes to the plowing force by influencing the hardness. Flowing by the micro-grinding force of the single grit proposed, the overall micro-grinding force of the whole wheel is extrapolated based on the grinding wheel geometry.
Table 1. Johnson-Cook parameters and constants for single-crystal copper used in equation (3) [25].

\begin{tabular}{lllll}
\hline$A(\mathrm{MPa})$ & $B(\mathrm{MPa})$ & $n$ & $C$ & $m$ \\
\hline 330 & 53.7 & 0.56 & 0.026 & 1.04 \\
\hline
\end{tabular}

\section{Materials characterization}

Copper is a face-centered cubic (FCC) material. Three single-crystal copper materials $((100),(110)$, and(111)) were prepared for the study with dimension $10 \times 10 \times 1$ $\mathrm{mm}^{3}$. Electron Back Scatter Diffraction (EBSD) was used to observe the microstructure. The CO of the three samples were analyzed by the TSL OIM Data connection 5 software and depicted by the OIM micrographs, which are shown in Figure 1.

Single-crystal copper shows anisotropy on the different cutting planes and cutting directions in micro-cutting. In the investigation, six cutting directions were chosen with two crystal directions of each sample tested using EBSD, as shown in Figure 2. In the investigation, CO is presented by Miller indices [24]. For example, $[h k l]$ is parallel to the grinding direction, while $\left(\begin{array}{lll}u & v & w\end{array}\right)$ is perpendicular to the grinding plane.

The Johnson-Cook parameters of single-crystal copper, which are referred to in reference [25], are listed in Table 1, and the additional material properties for modeling forces are provided in Table 2.

\section{Calculation methods}

\subsection{Flow stress calculations}

Flow stress includes thermally activated stress and athermal stress based on the dislocation theory [31], which is given by

$$
\sigma=\sigma_{0}+\sigma_{1}
$$

where the thermally activated stress $\sigma_{0}$ denotes the flow stress with a dislocation density of zero. Johnson-Cook model [32] considers the effects of strain, strain rate, and temperature on the shear plane, which is given by

$$
\sigma_{0}=\left(A+B_{\varepsilon}^{n}\right)\left(1+C \ln \frac{\dot{\varepsilon}}{\dot{\varepsilon}_{0}}\right)\left(1-\left(\frac{T_{0}-T_{w}}{T_{m}-T_{w}}\right)^{m}\right) .
$$

The computations of strain and strain rate in this work are calculated based on Oxley's model [33]. The athermal activation stress model was proposed by Hughes et al. [16], who represented the effect of dislocations as follows:

$$
\sigma_{1}=M \alpha_{1} G b_{1} \sqrt{\rho_{1}}
$$

where $M$ is the Taylor factor, $G$ is the shear modulus of material, $\alpha_{1}$ is the material constant, $b_{1}$ is the burger vector of dislocations, and $\rho_{1}$ is the total density of dislocation. The modified material model of micro-grinding is 


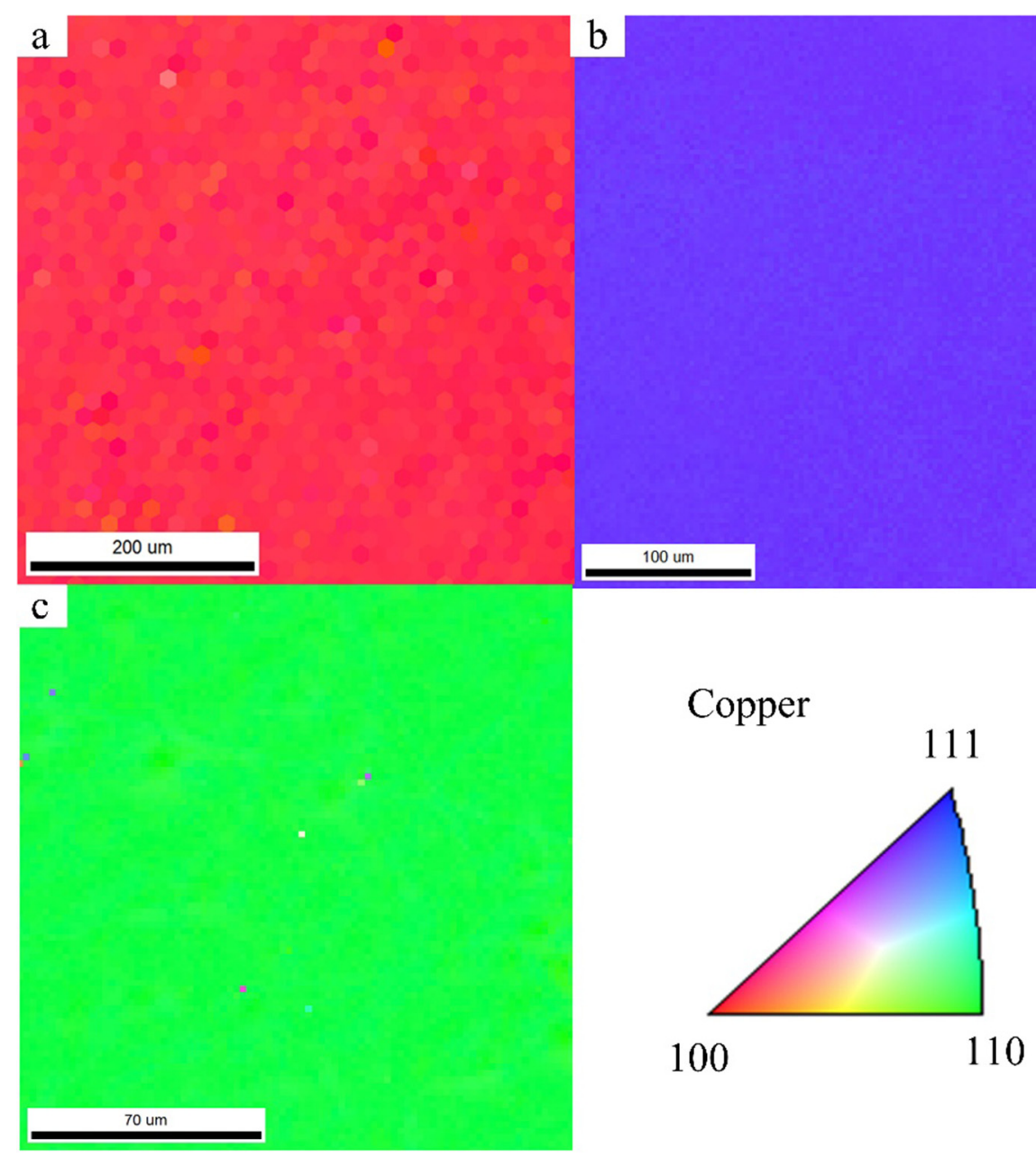

Fig. 1. The CO of the three single copper samples.
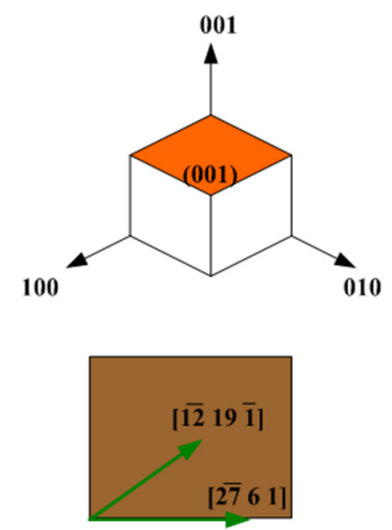

(a) (001)
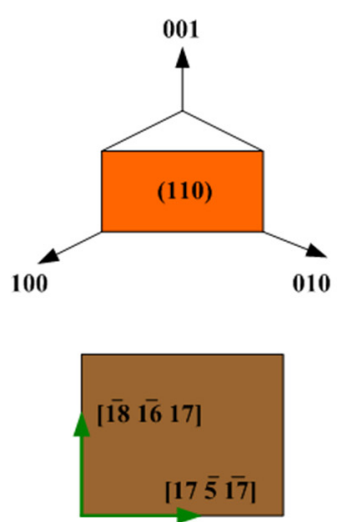

(b) (110)
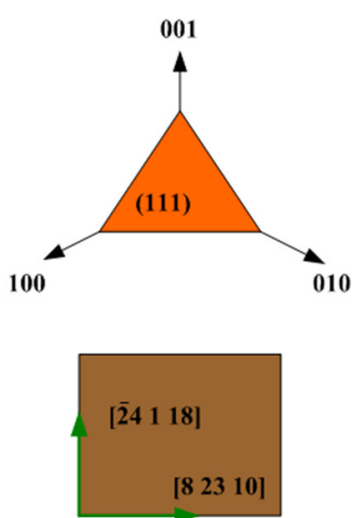

(c) (111)

Fig. 2. Cutting direction in the crystal coordinate system.

proposed as follows:

$$
\begin{aligned}
\sigma= & \left(A+B \varepsilon^{n}\right)\left(1+C \ln \frac{\dot{\varepsilon}}{\dot{\varepsilon}_{0}}\right)\left(1-\left(\frac{T_{0}-T_{w}}{T_{m}-T_{w}}\right)^{m}\right) \\
& +M \alpha_{1} G b_{1} \sqrt{\rho_{1}} .
\end{aligned}
$$

However, $M$ is taken as a constant of 3.06 in Hughes' model.

\subsection{Modeling Taylor factor}

Crystals with an FCC structure are inherently anisotropic and have a number of independent slip systems, which results in the active deformation textures. The activation condition of the slip systems plays a significant role in describing the variations of the plastically deformed zone, the plastic shears, and the dislocation density. Each crystal 
Table 2. Additional material properties used in the model.

\begin{tabular}{llll}
\hline Parameters & Description & Values & Reference \\
\hline$E$ & Modulus of elasticity & $129.8 \mathrm{MPa}$ & {$[26]$} \\
$v$ & Poisson's ratio & 0.343 & {$[26]$} \\
$\rho$ & Density & $8960 \mathrm{~kg} / \mathrm{m}^{3}$ & {$[27]$} \\
$\alpha$ & Thermal diffusivity & $18 \mathrm{E}-6{ }^{\circ} \mathrm{C}^{-1}$ & {$[26]$} \\
$C_{p}$ & Specific heats & $386 \mathrm{~J} / \mathrm{kg}{ }^{\circ} \mathrm{C}$ & {$[26]$} \\
$k$ & Thermal conductivities & $401 \mathrm{~W} / \mathrm{m}^{\circ} \mathrm{C}$ & {$[27]$} \\
$T_{\text {melt }}$ & Melting temperature & $1084.62{ }^{\circ} \mathrm{C}$ & {$[27]$} \\
$\alpha_{1}$ & Material constant & 0.24 & {$[28]$} \\
$b_{1}$ & Burgers vector & $0.308 \mathrm{~nm}$ & {$[29]$} \\
$\rho_{1}$ & Dislocation density & $5 \mathrm{E}-4 \mathrm{~nm}^{-2}$ & {$[30]$} \\
\hline
\end{tabular}

Table 3. The 12 slip systems of the FCC crystal.

\begin{tabular}{|c|c|c|c|c|c|c|c|}
\hline Plane & (111) & & & Plane & $(\overline{11} 1)$ & & \\
\hline Direction & {$[0 \overline{1} 1]$} & {$[01 \overline{1}]$} & {$[\overline{1} 10]$} & Direction & {$[011]$} & {$[101]$} & {$[1 \overline{1} 0]$} \\
\hline Plane & $(\overline{1} 11)$ & & & Plane & $(1 \overline{1} 1)$ & & \\
\hline Direction & {$[0 \overline{1} 1]$} & {$[\overline{1} 0 \overline{1}]$} & [110] & Direction & {$[011]$} & {$[10 \overline{1}]$} & {$[\overline{11} 0]$} \\
\hline
\end{tabular}

system owns a different number and style of slip systems. In the FCC crystals, the slip planes belong to the family $\{111\}$, while the slide direction belongs to the 011 family. According to the different unique planes and directions in these two families, the FCC system can be classified into 12 slip systems. The 12 slip systems of FCC lattice are summarized in Table 3 [34].

The Schmidt factor was proposed by Schmidt and Boas [35] to investigate the tensile yield stress in the tensile test of material, which is related to the COs of material. Moreover, $m_{c} m_{c}=1 /(\cos \Phi \cos \Omega)$ which is the reciprocal of the Schmidt factor of most orientation advantageous slip systems in grain, changes with the variation of the $\mathrm{CO}$ of the monocrystalline material. In the uniaxial drawing, the formula $M=m_{c}$ is built, and the calculated value of $m_{c}$ varies with different polycrystalline deformation models [36].

In this article, the following assumptions are made:

1. Taylor factor is proposed for the tensile test with low strain rate, nearly 10 ; this article assumes that it is valid in machining with high strain rate, nearly $10^{4}$.

2. The inverse of the Schmidt factor is referred as the Taylor factor, which is proposed for the tensile test in the single slip, this article assumes that it is valid in multislip.

3. This article assumes that the total Taylor factor of multi-slip is a linear additivity model of the activated individual Taylor factors.

During the micro-grinding process, the chip formation and plowing forces are caused by the material dislocation slip. The formula of the Taylor factor is as follows:

$$
M=\sum_{i=1}^{m} 1 /\left(\cos \left(\Phi_{i}+\varphi_{1}\right) \cos \left(\Omega_{i}+\varphi_{2}\right)\right),
$$

where $\Phi$ is the angle between the slip plane and cutting plane in grain, $\Omega$ is the angle between the slip direction and cutting direction in grain, $\varphi_{1}$ is the angle between the tangential plane and shear plane, $\varphi_{2}$ is the angle between the tangential direction and cutting direction and $m$ represents the total activated slip systems.

Specific energy can be obtained in terms of the Taylor factor, the shear angle, and the hardness, as follows:

$$
K_{c u t}^{s p}=\frac{M \tau_{c r}}{\tan \varphi}-\frac{H}{3}
$$

where $\varphi$ is the shear angle calculated using the parallelsided shear zone model.

\subsection{Modeling of mechanical effects in micro-grinding}

In modeling the individual grit interaction, the geometry of grits is simplified and the different shapes have been used to present the cutting edge such as pyramid by Badger and Torrance [37], cone by Law et al. [38], and sphere by Show [39]. The difference between these approximations is little since the cutting depth with respect to the grit diameter is so small [40]. The research assumes the abrasive grit shape to be a sphere. In addition, a mathematical framework for the individual grit interaction in micro-grinding is proposed involving micro-cutting, plowing, and rubbing components, as shown in Figure 3.

The tangential and normal forces of individual grit are obtained by integrating the infinitesimal elements, with the intermediate variable shear angle, the strain, and the strain rate calculated in terms of the slip line field theory [33]. Park and Liang [13] proposed the calculative method of 


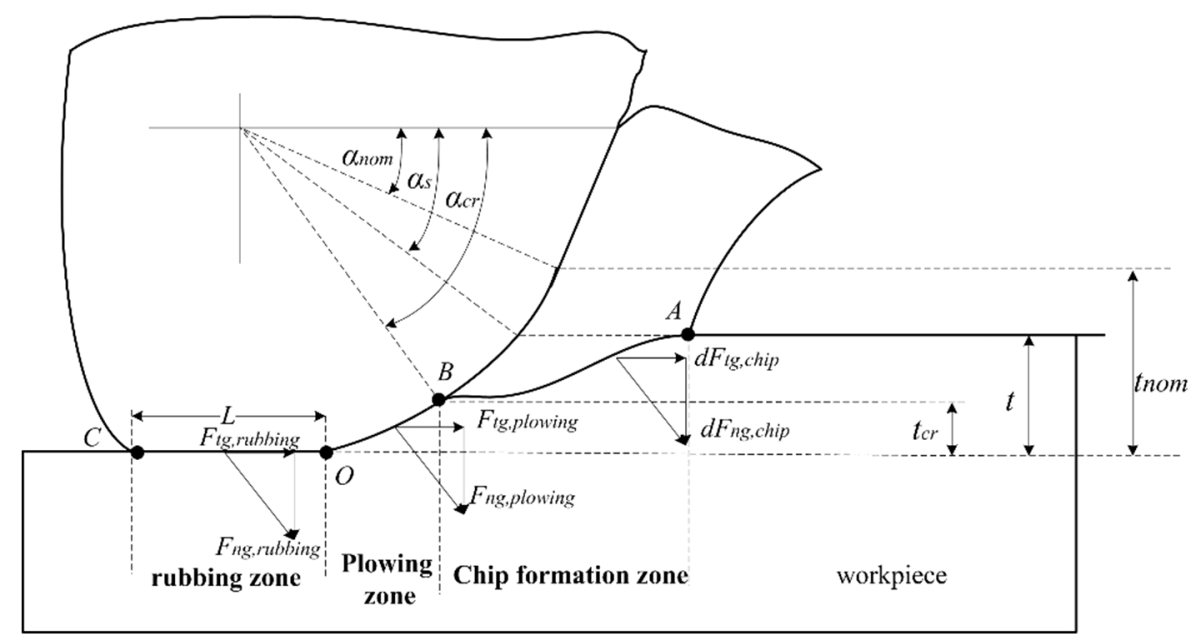

Fig. 3. Single grit interaction illustration.

chip formation and plowing forces, while the authors neglected the rubbing force at sliding contact between the workpiece and the grit.

The rubbing force results from the elastic or elasticplastic contact between grit wear flat area and the workpiece surface [41]. It is experimentally proved that the normal force varies with the wear area [42]. Shao [43] proposed an analytical approach to calculate the normal and shear stress in the wear flat area. The tangential force $F_{\text {tg,rubbing }}$ and the normal force $F_{\text {ng,rubbing }}$ are estimated by integrating the normal stress and shear stress, respectively:

$$
\left\{\begin{array}{l}
F_{t g, \text { rubbing }}=b \int_{0}^{l_{O C}} \sigma_{w} d x \\
F_{n g, \text { rubbing }}=b \int_{0}^{l_{O C}} \tau_{w} d x
\end{array}\right.
$$

where $b$ is the width of the grit,

$$
\begin{cases}\sigma_{w}(x)=\sigma_{0}\left(\left(l_{o c}-x\right) / l_{o c}\right)^{2} & \text { for } 0<x<l_{o c} \\ \tau_{w}(x)=\tau_{0} & \text { for } 0<x<l_{o c}\left(1-\sqrt{\tau_{0} / \sigma_{0}}\right), \\ \tau_{w}(x)=\mu \sigma_{w}(x) & \text { for } l_{\mathrm{oc}}\left(1-\sqrt{\tau_{0} / \sigma_{0}}\right)<x<l_{o c}\end{cases}
$$

For the individual grit, the sliding length of wear flat area could be calculated by equation (9) as

$$
l_{\mathrm{oc}}=2 r(1 / \cos (\theta / 2)-1) \tan (\theta / 2)
$$

where $r$ and $\theta$ are the radius and cone angle of individual grit, respectively.

In the process, the flow stress is related to the yield strength of material and the microstructure strengthening, which is presented by equation (10) as

$$
\sigma_{0}=A+M \alpha_{1} G b_{1} \sqrt{\rho_{1}},
$$

and $\tau_{0}$ is given by $\tau_{0}=\sigma_{0} / \sqrt{3}$.

\subsection{Modeling the wheel topography}

The overall micro-grinding force is calculated based on the shape of the grit edges and the wheel topography. Hacker [44] presented the static cutting edge density with equation (11)

$$
C_{s}=A_{s} Z^{k_{s}}
$$

where $C_{s}$ is the dynamic cutting edge density, $Z$ is the radial distance into the wheel, which is equal to the maximum undeformed chip depth, and $A_{s}$ and $k_{s}$ are the adjusted parameters related to the grit size obtained from the experiment. Various techniques such as profilometry, imprint method, scratch method, and microscopy have been developed to drive the topography of a grinding wheel. Hecker [44] provided the common relationship between static and dynamic cutting edge density.

The model of dynamic cutting edge density $C_{d}$ is presented in

$$
C_{d(Z)}=\frac{C_{s(z)}}{1+\frac{C_{s(z)}}{4} \frac{\tan (\theta)}{\tan \left(\varepsilon_{z}\right)} \frac{X^{3}}{Z}}
$$

where $\theta$ denotes the cone angle of grit, $\varepsilon_{z}$ is the infeed angle related to the dynamics of micro-grinding, and $\tan \left(\varepsilon_{z}\right)=$ $\frac{V_{w}}{V} \sqrt{\frac{a_{p}}{D_{w}}}$. The preceding cutting edge kinematically hides the next incoming small cutting edges, which makes a small portion of the cutting edges contact with the workpiece during grinding. The chip thickness is represented by the Rayleigh probability density function (p.d.f) $f(x, X)=$ $\left(x / X^{2}\right) \exp \left(-x^{2} / 2 X^{2}\right)$ [45], with the Rayleigh parameter $X=\sqrt{\frac{a_{p} V_{w}}{2 V l_{c} C_{d} \tan (\theta)}}$. The expected value and the variance for the p.d.f are $E(x)=1.253 X$ and $\operatorname{var}(x)=0.429 X^{2}$. The radial distance into the wheel $Z$ is represented as

$$
Z=E(x)+3 \sqrt{\operatorname{var}(x)} .
$$


Then, the total chip formation force is calculated as the multiplication of average single grit force and the number of active cutting edges,

$$
\left\{\begin{array}{l}
F_{t, \text { shear }}=C_{d} w l_{O B} F_{t g, \text { shearing }} \\
F_{n, \text { shear }}=C_{d} w l_{O B} F_{n g, \text { shearing }}
\end{array}\right.
$$

where the contact length of the shear plane $l_{\mathrm{AB}}$ is given by equation (15),

$$
l_{A B}= \begin{cases}0, & t \leq t_{c r}, \\ l_{c}-l_{O B}, & t_{c r} \leq t,\end{cases}
$$

where the total contact length $l_{c}$ is expressed as $l_{c}=$ $\left(a_{p} D_{e}+8 R_{r} F_{n g} D_{e}\left(K_{w}+K_{t}\right)\right)^{1 / 2}, a_{p}$ is the depth of cut, $D_{e}$ is the effective diameter, $K_{w}, K_{t}$ are the workpiece and wheel elasticity, respectively.

The contact length of plowing plane $l_{O B}$ is given by equation (16)

$$
l_{O B}= \begin{cases}r\left(\alpha_{c r}-\sin ^{-1} \frac{(t-r)}{r}\right), & t \leq t_{c r} \\ r\left(\alpha_{c r}-\alpha_{n o m}\right), & t_{c r}<t\end{cases}
$$

Similar to chip formation force, the total plowing and rubbing forces are also calculated as the multiplication of average single grit force and the number of active cutting edges. Then, the total grinding force is calculated as

$$
\left\{\begin{array}{l}
F_{t}=F_{t, \text { shearing }}+F_{t, \text { plowing }}+F_{t, \text { rubbing }} \\
F_{n}=F_{n, \text { shearing }}+F_{n, \text { plowing }}+F_{n, \text { rubbing }}
\end{array} .\right.
$$

\section{Experiment method}

To verify the assumption, the experiments of microgrinding single-crystal copper were conducted with the $\mathrm{CO}$ as the only variable and the micro-grinding parameters including wheel speed, federate, and the depth of cut as the constants. The arrangements of the inputs are listed in Table 4.

Micro-grinding experiments were conducted on a miniaturized machine tool without coolants as shown in Figure 4a. A Kistler 9256C2 MiniDyn was used to measure the forces. The configuration of the micro-grinding experiment is shown in Figure 4b. The experiments were carried out using a CBN grinding wheel 85412-BM with a diameter of $3 \mathrm{~mm}$.

The wheel topography of 85412-BM with 240-270 grit number was experimentally analyzed. The cutting edge radius $(r)$ and cone angle $(\psi)$ of abrasive grit were measured by the $3 \mathrm{D}$ digital microscope. Based on the experimental data presented by Zishan [46], a nonlinear regression analysis was performed to calculate the static cutting edge density with the constants $\left(A_{s}\right.$ and $\left.k_{s}\right)$ obtained. The topography parameters of $85412-\mathrm{BM}$ and the spindle specification are listed in Table 5.
Table 4. The parameters of the micro-grinding process in the single-factor experiment.

\begin{tabular}{ll}
\hline Parameters & Micro-grinding \\
\hline $\begin{array}{l}\text { Workpiece } \\
\text { material }\end{array}$ & Copper \\
$\begin{array}{l}\text { Tool edge } \\
\text { radius }\end{array}$ & $12 \mu \mathrm{m}$ \\
Depth of cut & $10 \mu \mathrm{m}$ \\
Wheel speed & $2.355 \mathrm{~m} / \mathrm{s}$ \\
Feed rate & $10 \mathrm{~mm} / \mathrm{min}$ \\
Workpiece & $(001)[\overline{27} 61](001)[\overline{12} 19 \overline{1}](110)[17 \overline{5} \overline{17}]$ \\
orientation & $(110)[\overline{1816} 17](111)[82310](111)[\overline{24} 118]$ \\
\hline
\end{tabular}

\section{Results and discussion}

\subsection{Calculated and experimental results}

The Taylor factor is calculated using equation (5) with the COs of samples provided in Figure 2. Then the flow stress is computed using equation (4) with the constants of Johnson-Cook and Hall-Petch equation provided by Tables 1 and 2. Strain and strain rate are calculated based on the methodology presented in reference [33].

The values of Taylor factor, shear angle, flow stress, and specific energy calculated in the procedure of predicting the micro-grinding force are listed in Table 6 . As an important mechanical property, Vickers hardness was measured by the digital Vickers hardness tester HVS-1000Z. The mean values of Vickers hardness of the six samples are also listed in Table 6.

Therefore, the chip formation and plowing forces of the single grit in the tangential and normal directions can be obtained by the model proposed in reference [23]. The rubbing force of single grit is calculated using equation (7). Then, the force model of the single grit interaction is extrapolated to the force model of the whole grinding wheel, based on the grinding wheel topography. Finally, micro-grinding forces in tangential and normal directions are calculated by equation (17).

Experimental data were collected from the six cases. Because the vibration of spindle rotating with high rpm generated noise in the force signal, a fast Fourier transform (FFT) analysis was performed for the output signals of the no micro-grinding region and the microgrinding region to identify the frequency range of the force signal, as displayed in Figure 5. Take Case 2 as an example. The results show that the vibration of the spindle rotating with $15,000 \mathrm{rpm}$ is around $264 \mathrm{~Hz}$, and the frequencies of the micro-grinding force signals are below $200 \mathrm{~Hz}$. To block the noise of the rotational spindle, all measurement signals were filtered with a low-pass filter, as shown in Figure 6. Then, the mean value and standard deviation of the tangential and normal forces are calculated. 


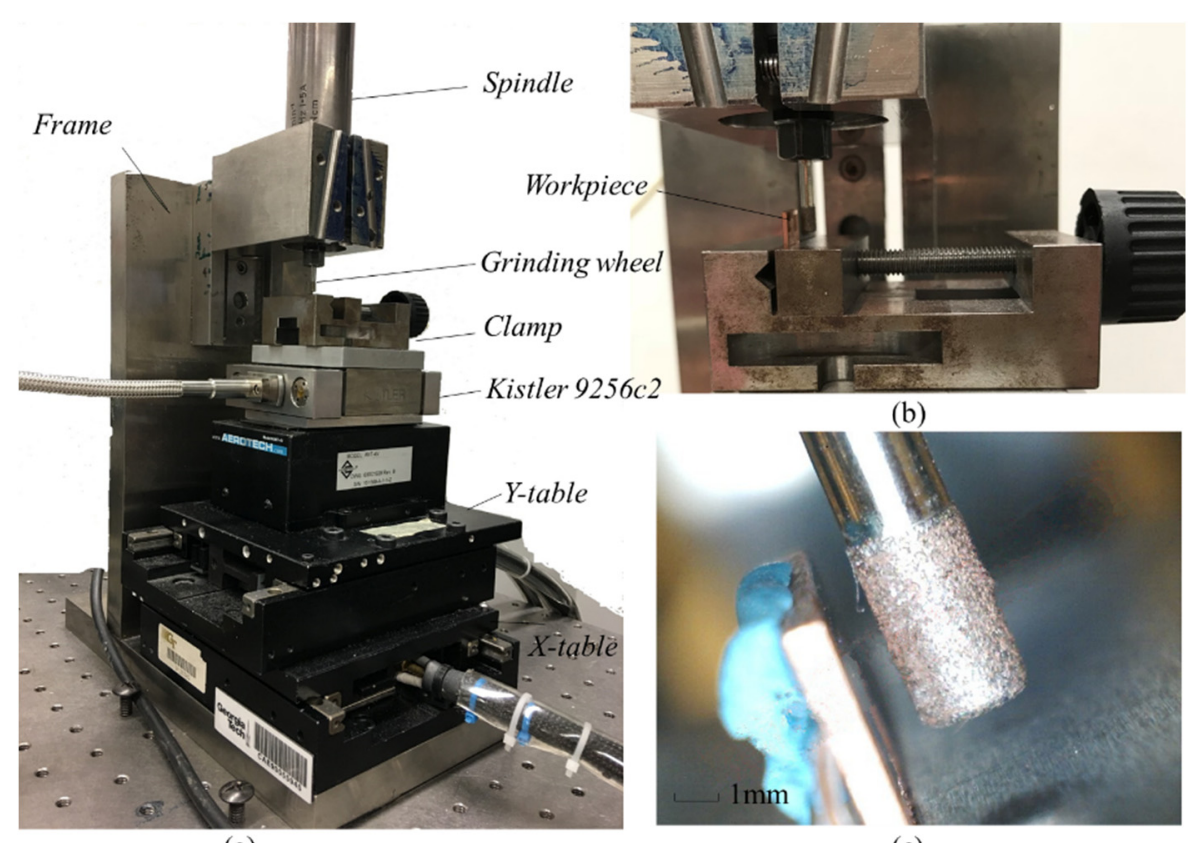

(a)

(c)

Fig. 4. Schematic of (a) a miniaturized machine tool, (b) an illustrated experimental configuration, and (c) an enlarged view of the micro-grinding process.

Table 5. The wheel topography parameters of 85412-BM and the spindle specification.

\begin{tabular}{lll}
\hline Item & Grinding spindle & Grinding wheel \\
\hline Specification & TYP: SLF Fraureuth FS33-60/0.15.1 & Cubic boron nitride \\
& Nr: 6939 & High-speed electroplated \\
& Voltage: $48 \mathrm{~V}$ & $A_{s}: 3.22$ \\
& Maximum rotating: $60000 \mathrm{~min}^{-1}$ & $K_{s}: 0.89$ \\
& Power: $170 \mathrm{~W}$ & $2 \mathrm{r}(\mathrm{um}): 26$ \\
& Moment: $6 \mathrm{~N} \mathrm{~cm}$ & $\psi[\mathrm{rad}]: 2.11$ \\
\hline
\end{tabular}

Table 6. The calculated Taylor factor, shear angle, flow stress, and specific energy.

\begin{tabular}{lllllll}
\hline Slip plane & $(001)$ & $(001)$ & $(110)$ & $(110)$ & $(111)$ & $(111)$ \\
Slip direction & {$[2761]$} & {$[\overline{12} 191]$} & {$[17517]$} & {$[181617]$} & {$[82310]$} & {$[24118]$} \\
\hline Taylor factor $M$ & 25.16 & 6.18 & 6.16 & 4.94 & 2.53 & 2.74 \\
Shear angle $\varphi(\mathrm{rad})$ & 0.51 & 0.51 & 0.51 & 0.51 & 0.51 & 0.51 \\
Flow stress $\sigma(\mathrm{MPa})$ & $7.79 \mathrm{e} 9$ & $7.67 \mathrm{e} 9$ & $5.89 \mathrm{e} 9$ & $2.82 \mathrm{e} 9$ & $6.85 \mathrm{e} 9$ & $4.51 \mathrm{e} 9$ \\
Vickers hardness $(\mathrm{HV})$ & 50.20 & 50.20 & 57.30 & 57.30 & 60.80 & 60.80 \\
Specific energy $K_{c u t}^{\text {sp }}(\mathrm{MPa})$ & $1.85 \mathrm{e} 5$ & $1.80 \mathrm{e} 5$ & $1.02 \mathrm{e} 5$ & $5.91 \mathrm{e} 3$ & $1.41 \mathrm{e} 5$ & $5.67 \mathrm{e} 5$ \\
\hline
\end{tabular}

\subsection{Model verification}

In order to analyze the effect of $\mathrm{CO}$ related to cutting direction on micro-grinding forces, the proposed force model considering the variation of Taylor factor is compared to the previously reported model in which
Taylor factor was taken as the constant of 3.06. The comparison between the predictions and the experimental data is shown in Figure 7 , where $F_{t}$ and $F_{n}$ refer to tangential and normal force respectively.

The force model considering the variation of Taylor factor performs well in terms of capturing the trend and 


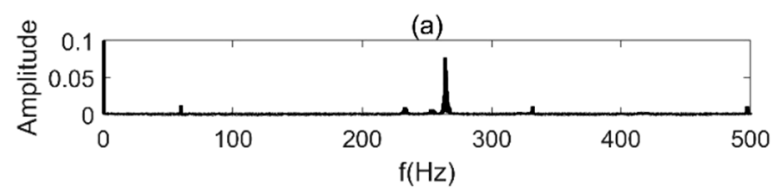

(b)
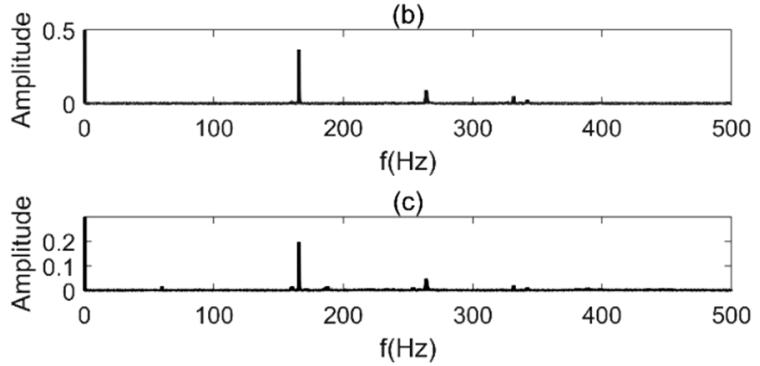

Fig. 5. FFT analysis for the output signal of (a) no microgrinding region and (b) micro-grinding region in the microgrinding process.

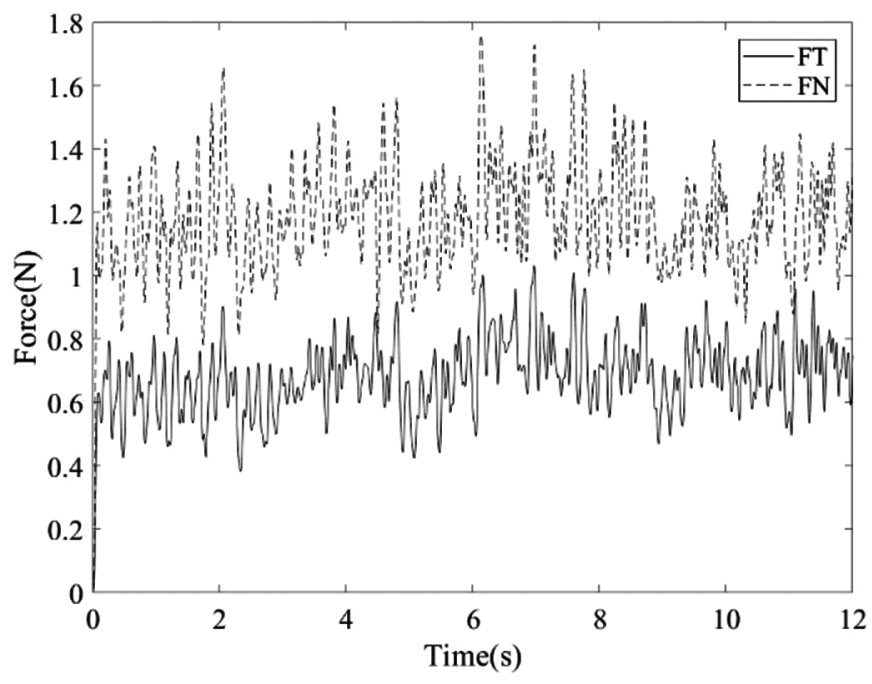

Fig. 6. Experimental data of micro-grinding after filtering.

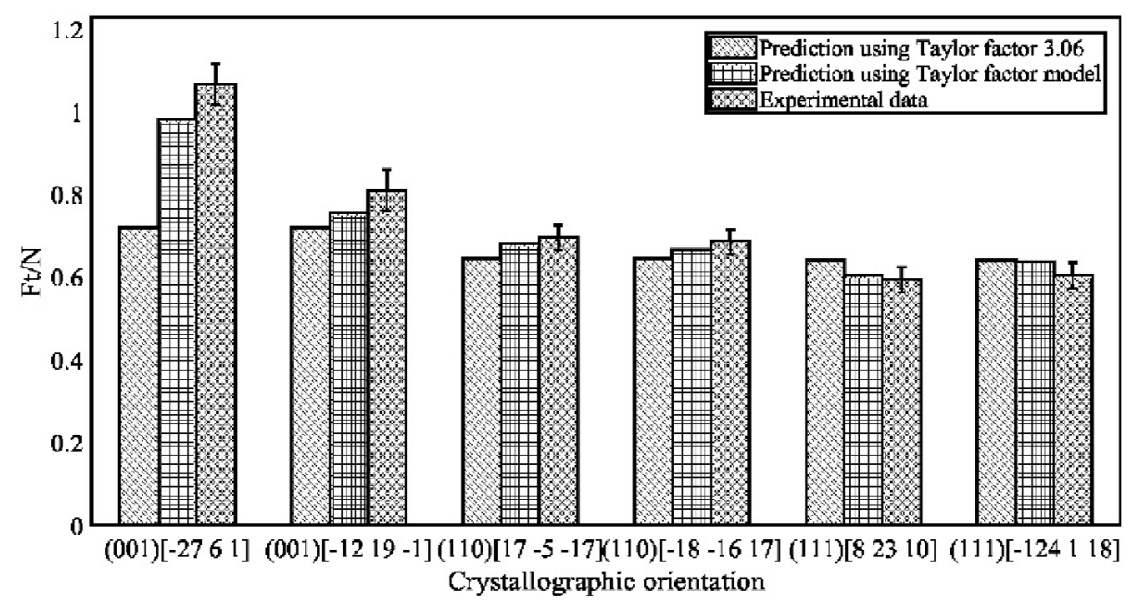

(a) Tangential force $F_{t}$

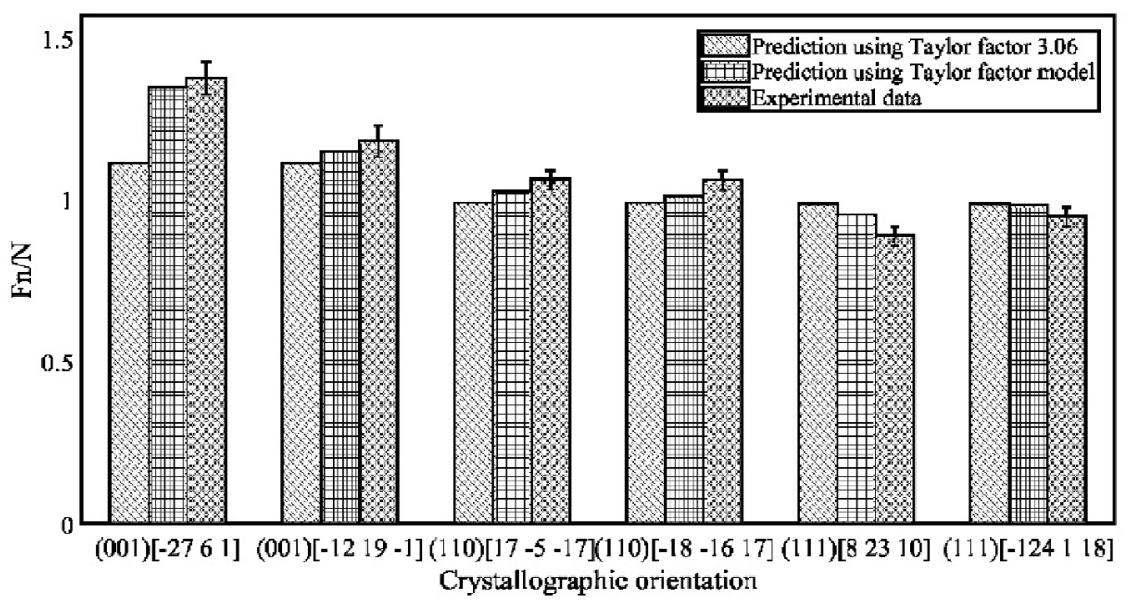

(b) Normal forces $F_{n}$

Fig. 7. The comparisons between predictions and experimental data. 


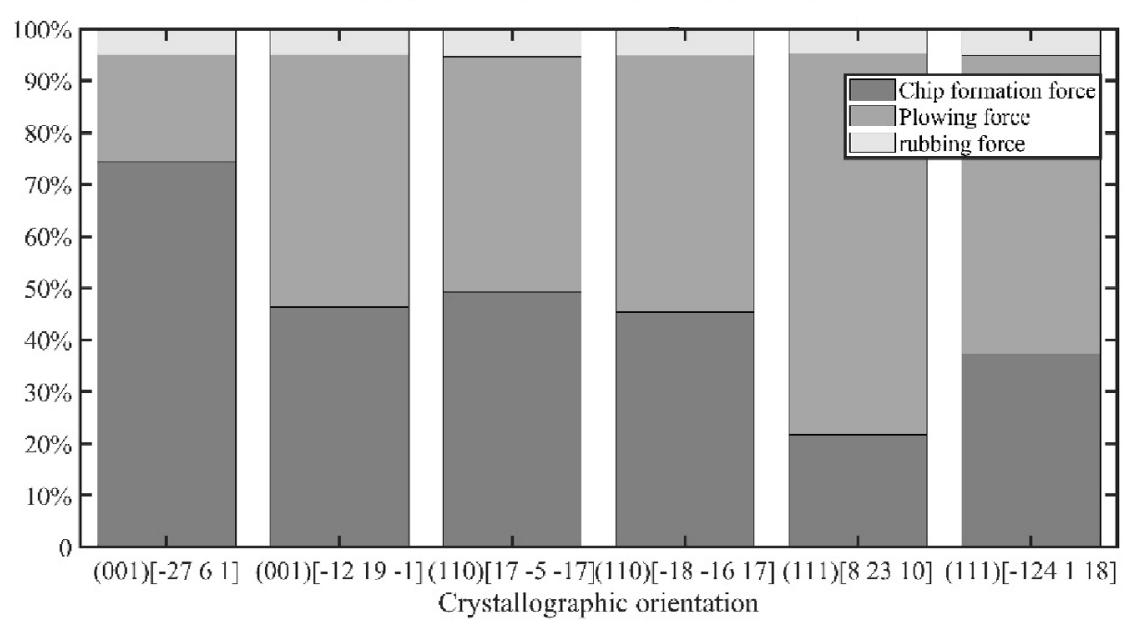

(a) Contribution of forces in tangential direction

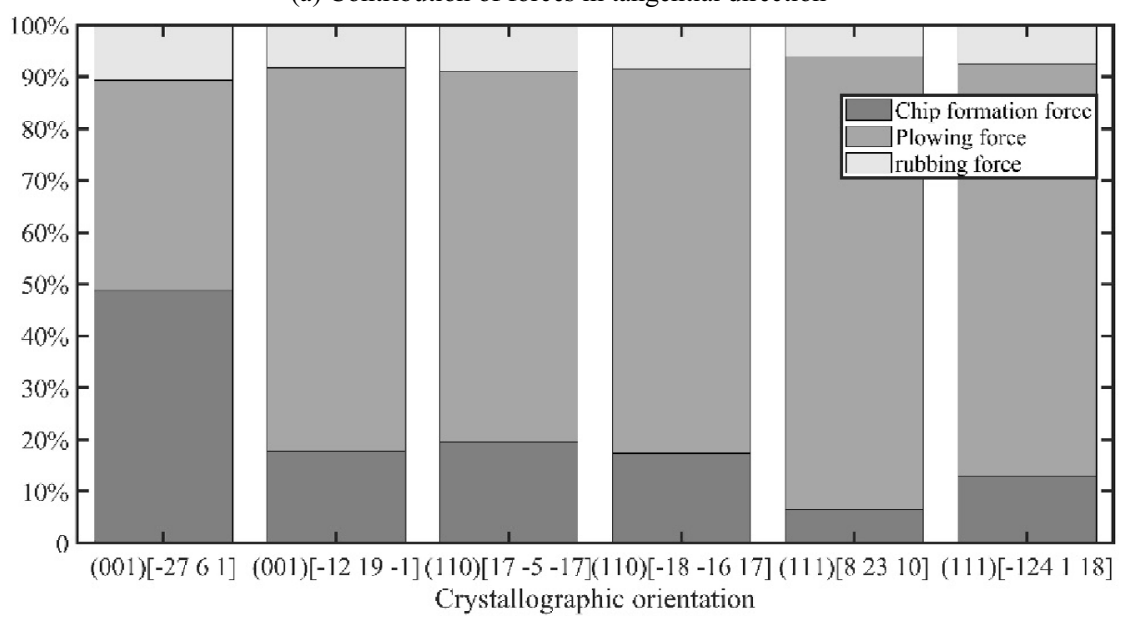

(b) Contribution of forces in normal direction

Fig. 8. The contribution of grinding forces in tangential and normal directions.

magnitude of the experimental data, with errors less than $8 \%$ in the tangential direction and $7 \%$ in the normal direction. The error bars representing the standard deviation indicate that the error between predictions and experiments is acceptable. The forces predicted by the model considering the Taylor factor as a constant of 3.06 does not capture the trend of the experimental data with the varying $\mathrm{CO}$ and cutting direction. The maximum errors between the measured data and predictions exceeds $32 \%$ in the tangential direction and $19 \%$ in the normal direction. It is found that the two forces predicted on the same plane are the same in different cutting directions using the model considering the Taylor factor as 3.06. By comparing the errors of the two predictions, it is drawn that the proposed force model, which considers the variation of Taylor factor, is able to improve the accuracy of prediction. Moreover, it is concluded that the proposed Taylor factor is reasonable in the quantitative analysis of COs. Thereby, the assumption made for the Taylor factor was verified.

From the analysis above, it is found that the forces on the cutting plane of (111) are the minimum both on tangential and normal direction biggest, the cutting plane of (110) are the medium, and the cutting plane of (100) are the maximum.

To understand the machining mechanism, it is necessary to analyze the components and the corresponding contributions to micro-grinding force. Figure 8 shows the contributions of three components to the forces in both normal and tangential directions.

In the micro-grinding condition, the chip formation, plowing, and rubbing forces are the contributors to both forces in tangential and normal directions. The results also show that the contributions vary with the Taylor factors. When the Taylor factor is large enough, the effect of chip formation provides the primary contribution to both forces, while the contribution to normal force is less than that in tangential force. When the Taylor factor is small enough, the effect of plowing provides the primary contribution to both forces. Moreover, the normal force is affected by plowing and rubbing forces more significantly than tangential force. In the calculations, the plowing force is related to the hardness of material while the chip formation and rubbing forces are affected by the Taylor 


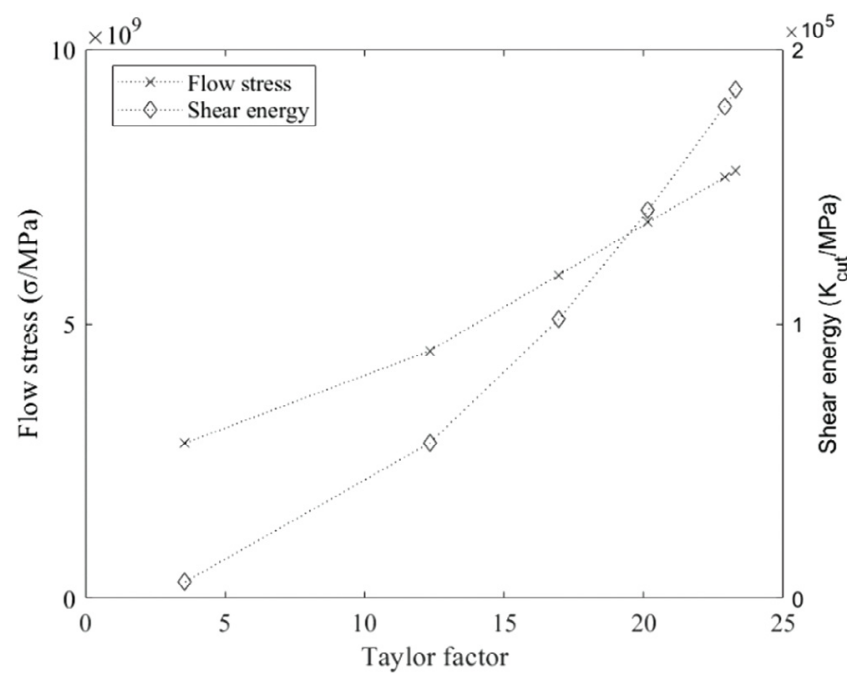

Fig. 9. The relationship between Taylor factor and flow stress as well as specific energy.

factor. In the mechanism, the anisotropy of the monocrystalline material is determined by the COs. In other words, the hardness is related to $\mathrm{CO}$ in the normal direction and the Taylor factor is related to $\mathrm{CO}$ in the tangential direction. The data listed in Table 6 show that the hardness increases when the Taylor factor decreases for different cutting planes. Therefore, the contributions vary with the Taylor factors.

\subsection{Sensitivity analysis}

Figure 9 depicts the relationship between the Taylor factors and the flow stress as well as specific energy resulting from the proposed model, which suggests that the Taylor factor has a significant positive correlation with the flow stress as well as specific energy.

Typically, the slip is restricted to a few of (symmetryrelated) slip systems consisting of a slip plane and a slip direction, and it generally occurs when the local stress projected to the plane exceeds a critical value. Besides, the activation of one slip system depends on the cutting direction, and the initially activated slip system(s) may show single-slip or multi-slip activity. The plastic slip activity in the full model is defined as the sum of the absolute values of the plastic slip along all slip systems. If the resistance to this sliding is very high, the slip system with high $M$ will not be activated. Take one case for example, when the tool grinding on the (100) surface, and the slip plane is (111), the relationship between the Taylor factor $(M)$ and the angle $(\Omega)$ between the cutting direction and slide direction is displayed in Figure 10.

\section{Conclusions}

This article provided an analytical model to predict microgrinding forces, which includes the chip formation, plowing, and rubbing forces, by considering the process parameters, wheel properties, and microstructure of

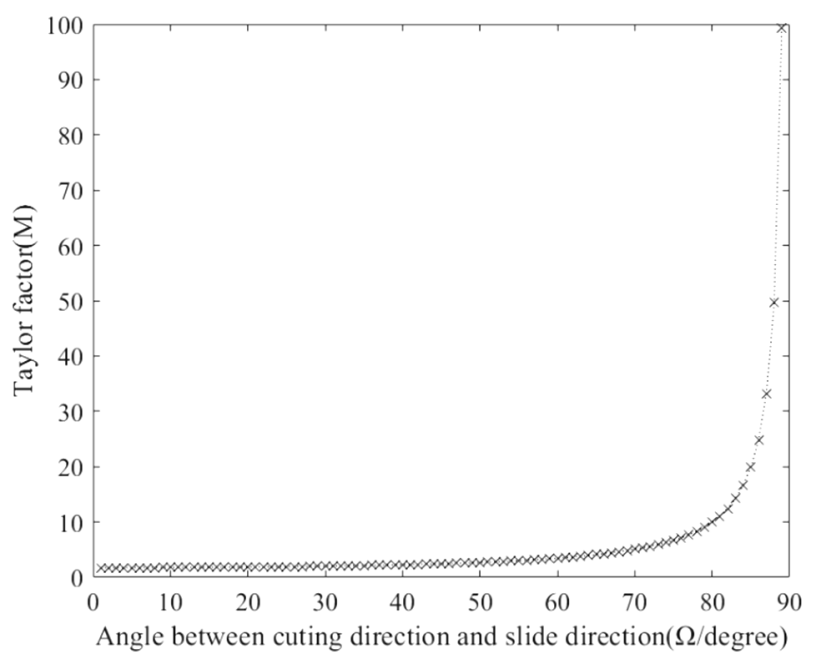

Fig. 10. The influence of CO on Taylor factor.

the workpiece material. The Taylor factor model was developed to estimate the number and style of the activated slip system(s), which are determined by the $\mathrm{CO}$ of the grinding plane and cutting direction in plastic deformation under some assumptions. Then, the flow stress and specific energy models were derived from the Taylor factor model.

The proposed force model was validated by conducting micro-grinding experiments and compared to the model considering the Taylor factor as a constant. The result showed that the proposed model considering the variability of Taylor factor is able to capture the trend and magnitude of the experimental data with the varying $\mathrm{CO}$ and the cutting direction, and improves the accuracy of the force model. Meanwhile, the assumptions proposed in this article are verified: the Taylor factor model is valid in machining with high strain rate, taking the inverse of the Schmidt factor as the Taylor factor is effective in multi-slip, and Taylor factor of multi-slip is a linear additivity model of the activated individual Taylor factors.

The sensitivity analysis showed that the flow stress and the specific energy in micro-grinding single copper are affected by $\mathrm{CO}$ with respect to cutting direction significantly. The contributions of three components to the total force indicate that the mechanism is related to the $\mathrm{CO}$ of material. It can be drawn that the plowing effect plays a much significant role in the micro-grinding process. The reason for the prominent plowing effect of the microgrinding can be attributed to the size effect.

The authors would like to gratefully acknowledge the equipment and support from the Precision Machining Research Center (PMRC), which is part of the Georgia Tech Manufacturing Institute (GTMI) at the Georgia Institute of Technology. We would also like to acknowledge National Natural Science Foundation of China (Grant No. 51705073) and Fundamental Research Funds for Central Universities of China (CUSF-DH-D2017060). 


\section{References}

1. D.A. Lucca, Y.W. Seo, R. Komanduri, Effect of tool edge geometry on energy dissipation in ultraprecision machining, CIRP Annals 42 (1993) 83-86

2. N. Fang, Slip-line modeling of machining with a roundededge tool: Part I: new model and theory, J. Mech. Phys. Solids 51 (2003) 715-742

3. M. Masuko, Fundamental research on the metal cuttingsecond report, Bull. Japan Soc. Mech. Eng. 118 (1956) 371

4. P. Abrecht, New developments in the theory of the metalcutting process, J. Eng. Ind 82 (1960) 348

5. M.C. Shaw, The size effect in metal cutting, Sadhana 28 (2003) 875-896

6. K. Nakayama, K. Tamura, Size effect in metal-cutting force, J. Eng. Ind. 90 (1968) 119-126

7. A.G. Atkins, Modelling metal cutting using modern ductile fracture mechanics: quantitative explanations for some longstanding problems, Int. J. Mech. Sci. 45 (2003) 373-396

8. S. Subbiah, S.N. Melkote, The size-effect in micro-cutting at low and high rake angles, Proceedings of 2004 ASME International Mechanical Engineering Congress and Exposition (IMECE04), 2004, pp. 485-493

9. T. Zhang, Z.Q. Liu, Z.Y. Shi, C.H. Xu, Investigation on size effect of specific cutting energy in mechanical micro-cutting, Int. J. Adv. Manuf. Technol. 91 (2017) 2621-2633

10. N. Chen, M.J. Chen, C.Y. Wu, X.D. Pei, Cutting surface quality analysis in micro ball end-milling of KDP crystal considering size effect and minimum undeformed chip thickness, Precis. Eng. 50 (2017) 410-420

11. B. Liu, F.Z. Fang, R. Li, Z.W. Xu, Y.S. Liang, Experimental study on size effect of tool edge and subsurface damage of single crystal silicon in nano-cutting, Int. J. Adv. Manuf. Technol. 98 (2018) 1093-1101

12. M.C. Shaw, New developments in grinding, Carnegie Press, Pittsburgh, 1972

13. H.W. Park, S.Y. Liang, Force modeling of micro-grinding incorporating crystallographic effects, Int. J. Mach. Tools Manuf. 48 (2008) 1658-1667

14. E.O. Hall, The deformation and ageing of mild steel: III discussion of results, Proc. Phys. Soc. B 64 (1951) 747-753

15. N.J. Petch, The cleavage strength of polycrystals, J. Iron Steel Inst. 174 (1953) 25-28

16. D.A. Hughes, N. Hansen, Microstructure and strength of nickel at large strains, Acta Mater. 48 (2000) 2985-3004

17. S.M. Byon, Y. Lee, Deformation analysis of micro-sized material using strain gradient plasticity, J. Mech. Sci. Technol. 20 (2006) 621-633

18. K. Ueda, K. Iwata, Chip formation mechanism in single crystal cutting of $\beta$-brass, CIRP Ann. Manuf. Technol. 29 (1980) 41

19. X. Wu, L. Li, N. He, M. Zhao, Z. Zhan, Investigation on the influence of material microstructure on cutting force and bur formation in the micro cutting of copper, Int. J. Adv. Manuf. Technol. 79 (2015) 321-327

20. B.L. Lawson, N. Kota, O.B. Ozdoganlar, Effects of crystallographic anistropy on orthogonal micromachining of single-crystal aluminum, J. Manuf. Sci. Eng. 130 (2008) 031116

21. J.Z. Li, B. Song, S.F. Wen, Y.S. Shi, A new insight of the relationship between crystallographic orientation and microcracks of Ti-47Al-2Cr-2Nb alloy, Mater. Sci. Eng. A 731 (2018) 156-160
22. F. Xu, F. Fang, Y. Zhu, X. Zhang, Study on crystallographic orientation effect on surface generation of aluminum in nanocutting, Nanoscale Res. Lett. 12 (2017) 289

23. M. Zhao, X. Ji, B. Li, S.Y. Liang, Investigation on the influence of material crystallographic orientation on grinding force in the micro-grinding of single-crystal copper with single grit, Int. J. Adv. Manuf. Technol. 90 (2016) 3347-3355

24. W. Tayon, R. Crooks, M. Domack, J. Wagner, A.A. Elmustafa, EBSD study of delamination fracture in $\mathrm{Al}-\mathrm{Li}$ alloy2090, Exp. Mech. 50 (2008) 135-143

25. B.Y. Cao, D.H. Lassila, M.S. Schneider, B.K. Kad, C.X. Huang, Y.B. Xu, D.H. Kalantar, B.A. Remington, M.A. Meyers, Effect of shock compression method on the defect substructure in monocrystalline copper, Mater. Sci. Eng. A 409 (2005) 270-281

26. K.J. Frutschy, R.J. Clifton, High-temperature pressureshear plate impact experiments on OFHC copper, J. Mech. Phys. Solids 46 (1998) 1723-1744

27. C. MTI, Available at http://www.Mtixtl.Com/cucrystalsub stratessinglecrystal.Aspx, 2018

28. H. Conrad, Grain-size dependence of the flow stress of $\mathrm{Cu}$ from millimeters to nanometers, Metall. Mater. Trans. A 35A (2004) 15

29. T. Suzuki, I.I. Yonenaga, H.O. Kirchner, Yield strength of diamond, Phys. Rev. Lett. 75 (1995) 3470-3472

30. M.R. Staker, D.L. Holt, The dislocation cell size and dislocation density in copper deformed at temperatures between 25 and $700^{\circ} \mathrm{C}$, Acta Metall. 20 (1972) 569-579

31. F.H. Abed, G.Z. Voyiadjis, Plastic deformation modeling of AL-6XN stainless steel at low and high strain rates and temperatures using a combination of bcc and fcc mechanisms of metals, Int. J. Plast. 21 (2005) 1618-1639

32. G.R. Johnson, W.H. Cook, A constitutive model and data for metals subjected to large strains, high strain rates and high temperatures, Proceedings of the Seventh International Symposium on Ballistics, The Hague, 1983, pp. 541-547

33. P.L.B. Oxley, The mechanics of machining: an analytical approach to assessing machinability, Halsted Press New York, UK, 1989

34. S.A. Tabei, Modeling of microstructural evolutions in machining of dual phase alloys, Georgia Institute of Technology, Atlanta, USA, 2015

35. E. Schmidt, W. Boas, Plasticity of crystal, Springer, Berlin, 1950

36. L. Cong, China metallurgical encyclopedia, metal and plastic processing, Metallurgical Industry Press, Beijing, China, 1999

37. J.A. Badger, A.A. Torrance, A comparison of two models to predict grinding forces from wheel surface topography, Int. J. Mach. Tools Manuf. 40 (2000) 1099-1120

38. S.S. Law, S.M. Wu, A.M. Joglekar, On building models for the grinding process, J. Eng. Ind. 95 (1973) 983-991

39. M.C. Shaw, New developments in grinding, Carnegie Press, Pittsburgh, 1972

40. R.L. Hecker, S.Y. Liang, X.J. Wu, P. Xia, D.G.W. Jin, Grinding force and power modeling based on chip thickness analysis, Int. J. Adv. Manuf. Technol. 33 (2006) 449-459

41. S. Malkin, Grinding technology: theory and applications of machining with abrasives, Ellis Horwood Series in Mechanical Engineering, American Society of Civil Engineers, Reston, VA, 1989

42. L. Lichun, F. Jizai, J. Peklenik, A study of grinding force mathematical model, CIRP Annals 29 (1980) 245-249 
43. Y. Shao, Predictive modeling of residual stress in MQL grinding and surface characteristcs in grinding of ceramics, Georgia Institute of Technology, Atlanta, USA, 2015

44. R.L. Hecker, Part surface roughness modeling and process optimal control of cylindrical grinding, Georgia Institute of Technology, Atlanta, USA, 2003
45. M.A. Younis, H. Alawi, Probabilistic analysis of the surface grinding process, Trans. Canadian Soc. Mech. Eng. 8 (1984) 208-213

46. D. Zishan, Research on surface integrity and process optimal criterion of micro-grinding, Donghua University, Shanghai, China, 2016

Cite this article as: Man Zhao, Xia Ji, Beizhi Li, Steven Y. Liang, Forces prediction in micro-grinding single-crystal copper considering the crystallographic orientation, Manufacturing Rev. 5, 15 (2018) 